\title{
Substitution of Cytosine with Guanylurea Decreases the Stability of i-Motif DNA
}

\author{
Elisé P. Wright, ${ }^{\dagger}$ Katarzyna Lamparska, ${ }^{\dagger}$ Steven S. Smith, ${ }^{\ddagger}$ and Zoë A. E. Waller*, ${ }^{*}, \S \odot$ \\ ${ }^{\dagger}$ School of Pharmacy, University of East Anglia, Norwich Research Park, Norwich NR4 7TJ, U.K. \\ ${ }^{*}$ Beckman Research Institute and Division of Urology, City of Hope, 1500 East Duarte Road, Duarte, California 91010-3000, United \\ States \\ ${ }^{\S}$ Centre for Molecular and Structural Biochemistry, University of East Anglia, Norwich Research Park, Norwich NR4 7TJ, U.K.
}

\section{Supporting Information}

ABSTRACT: Both 5-aza-2'-deoxycytidine (decitabine) and its primary breakdown product, $2^{\prime}$-deoxyriboguanylurea (GuaUre-dR), have been shown to act as mutagens and epimutagens that cause replication stress and alter both DNA methylation and gene expression patterns. As cytosine analogues, both are expected to be preferentially incorporated into regions of GC skew where runs of cytosine residues are sequestered on one strand and guanine residues on the other. Given that such regions have been identified as sites with the potential for effects on gene expression and replication stress linked to formation of alternative DNA secondary structures, it is of interest to determine the influence that these base analogues might have on the stability of structures of this kind. Here we report that incorporation of GuaUre-dR into an i-motif-forming sequence decreases both the thermal and $\mathrm{pH}$ stability of an i-motif despite the apparent ability of GuaUre$\mathrm{dR}$ to base pair with cytosine.

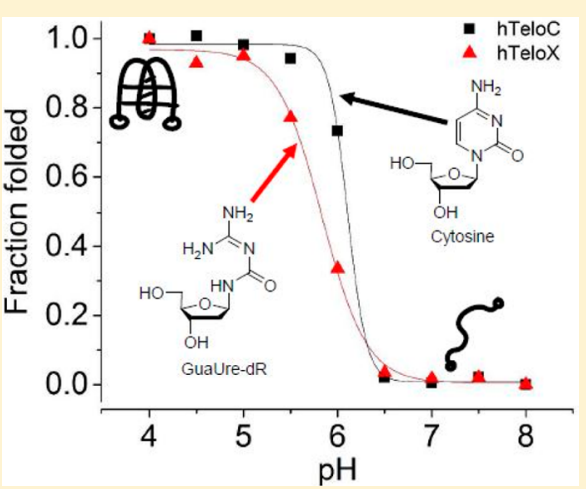

ferases, inducing hypomethylation and encouraging DNA instability, much like its precursor.,

5azaC-dR has been shown to induce the proteolytic degradation of DNMT1 but not of DNMT3a or DNMT3b. While the formation of a covalent bond between DNMT1 and 5azaC-dR-treated DNA could be ruled out as a cause of proteolysis, the mechanism by which DNMT1 is targeted for degradation is not yet well understood. ${ }^{6}$ However, it is known that noncanonical DNA structures rich in cytosine are targeted by DNMT1. ${ }^{7}$ This interaction results in methylated CG dinucleotides at sites of abnormal base pairing that bind to and trap the DNMT1 enzyme in tight DNA complexes. ${ }^{8-11}$ An interaction with a noncanonical DNA structure, such as a hairpin or an i-motif (C-quadruplex), could trap the enzyme and provide an additional trigger for the proteolysis of DNMT1. Cytosine-rich i-motif and hairpin structures are dependent on hemiprotonated cytosine:cytosine base pairing, ${ }^{12}$ and a single sequence can give rise to either morphology or both in equilibrium depending on the environment. ${ }^{13-15}$ It has been proposed that GuaUre-dR can base pair with cytosine in the absence of protonation; ${ }^{16}$ this suggests that the presence of GuaUre-dR in a cytosine-rich sequence may influence the stability and type of secondary structure that may form. The human telomeric i-motif sequence is a well-characterized model oligonucleotide that has been extensively used as a typical i-

Received: July 4, 2017

Revised: August 8, 2017

Published: August 30, 2017 


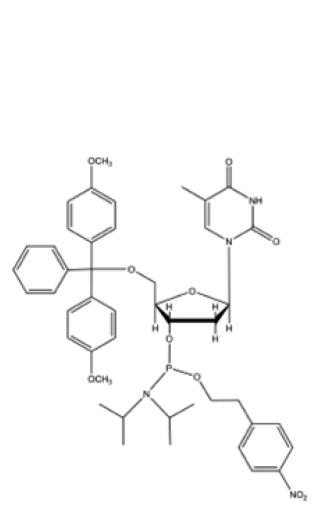

1

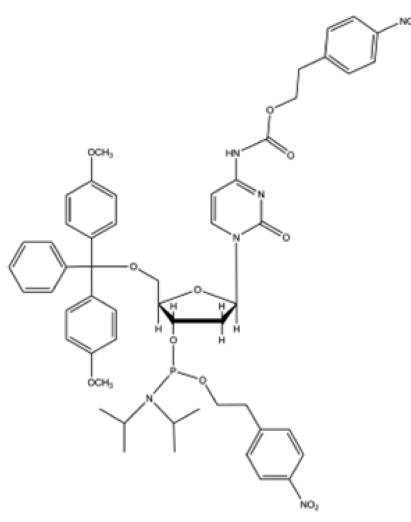

2

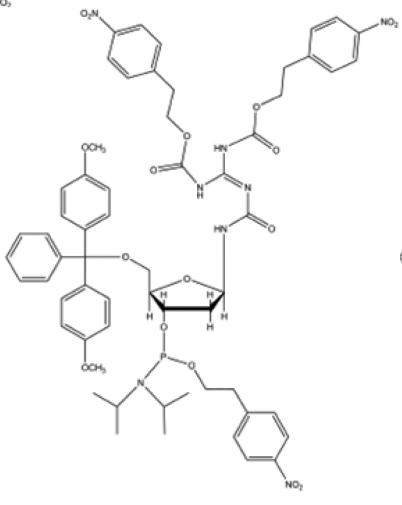

3

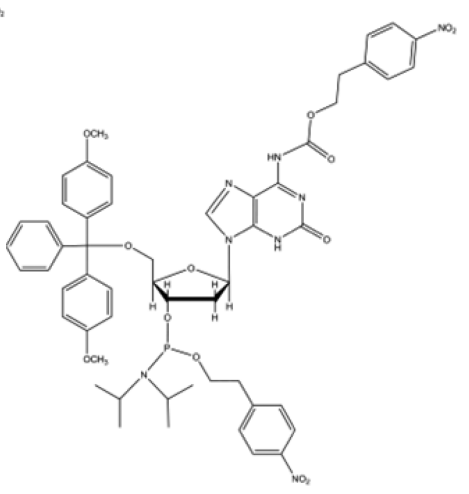

4

Figure 2. Structures of phosphoramidites prepared for use in the synthesis of the GuaUre-dR-containing oligodeoxynucleotide: 1 , $5^{\prime}$-Odimethoxytrityl-3'-O-[2-(4-nitrophenyl)ethoxy- $N, N$-diisopropyl]-2'-deoxythymidine phosphoramidite; 2, 5'-O-dimethoxytrityl-3'-O-[2-(4nitrophenyl)ethoxy- $N, N$-diisopropyl]-2'-deoxy-N4-[2-(4-nitrophenyl)ethoxycarbonyl]cytidine phosphoramidite; 3, 5'-O-dimethoxytrityl-3'-O-[2(4-nitrophenyl)ethoxy- $N, N$-diisopropyl]-2'-deoxyribofuranosyl-3-guanyl- $N, N$-bis[2-(4-nitrophenyl)ethoxycarbonyl] urea phosphoramidite; 4, 5'-Odimethoxytrityl-3'-O-[2-(4-nitrophenyl)ethoxy- $N, N$-diisopropyl]-2'-deoxy-N6-[2-(4-nitrophenyl)ethoxycarbonyl]adenosine phosphoramidite. Each phosphoramidite was characterized prior to use by chromatography, ${ }^{31} \mathrm{P}$ nuclear magnetic resonance, and mass spectrometry as described previously. ${ }^{3}$

motif structure. ${ }^{17}$ This sequence was modified with GuaUre-dR to determine its effect on the stability of the resultant secondary structure. Ultraviolet (UV) thermal melting, thermal difference spectra, and circular dichroism spectroscopy were used to characterize the $\mathrm{pH}$ and thermal stability of the human telomeric i-motif sequence with and without GuaUre-dR modification.

\section{MATERIALS AND METHODS}

Oligonucleotide Synthesis. The methods used in the synthesis of oligodeoxynucleotides and associated phosphoramidites have been described in detail. ${ }^{3}$ All syntheses were performed on a $1 \mu \mathrm{mol}$ scale using a Cyclone Plus DNA Synthesizer (MilliGen Bioresearch, Novato, CA). In brief, the syntheses were performed as follows.

Human Telomeric Sequence (hTeloC). Synthesis of the control sequence was performed using phosphoramidites purchased from Glen Research (Sterling, VA). Deprotection was performed with $32 \%$ ammonium hydroxide overnight at 55 ${ }^{\circ} \mathrm{C}$, followed by purification using high-performance liquid chromatography (HPLC) as described previously. ${ }^{3}$ The resulting pure fractions were characterized by polyacrylamide gel electrophoresis and mass spectrometry. The control 24-mer [5'-TAA-CCC-TAA-CCC-TAA-CCC-TAA-CCC-3' (hTeloC)] had a predicted monoisotopic mass (MS) of 7127.246. The observed MS was 7127.274, and the predicted MW was $7130.723 \mathrm{~g} \mathrm{~mol}^{-1}$. The yield of the pure oligodeoxynucleotide was $2.12 \mathrm{mg}(297.3 \mathrm{nmol})$.

Modified Human Telomeric Sequence (hTeloX, X = GuaUre). As previously noted, ${ }^{3}$ GuaUre-dR-containing oligodeoxynucleotides are not stable when deprotection is performed with standard ammonium hydroxide. Consequently, we adopted an alternative approach using $p$-nitrophenylethoxycarbonyl protecting groups for this purpose., ${ }^{3,18}$ The required phosphoramidites were synthesized and characterized as previously described. ${ }^{3}$ Each phosphoramidite carried a dimethoxytrityl group at the $5^{\prime}$ coupling site and a bis $(\mathrm{N}, \mathrm{N}$-didisopropylamino)-2-(4-nitrophenyl) ethoxyphosphane group at the $3^{\prime}$ coupling site. Primary amines were protected with $p$-nitrophenylethoxycarbonyl groups (Figure 2).

Synthesis of the homologous GuaUre-dR-containing sequence with GuaUre-dR $=\mathrm{X}$ at position 6 was performed using phosphoramidites depicted in Figure 2. This position was selected as previous work $^{19}$ has shown that epigenetic modification of the cytosines adjacent to a loop changed the stability of the structure. Deprotection was performed with a solution of $0.5 \mathrm{M}$ 1,8-diazabicyclo[5.4.0] undec-7-ene in anhydrous pyridine containing $5 \mathrm{mg} / \mathrm{mL}$ thymine for $16 \mathrm{~h}$ at room temperature ${ }^{20}$ as previously described. ${ }^{3}$ Deprotection was followed by purification using HPLC as previously described. ${ }^{3}$ The resulting pure fractions were characterized by polyacrylamide gel electrophoresis and mass spectrometry. The GuaUredR-containing 24-mer [5'-TAA-CCX-TAA-CCC-TAA-CCCTAA-CCC-3' (hTeloX)] had a predicted monoisotopic mass (MS) of 7118.257. The observed MS was 7118.268, and the predicted MW was $7121.713 \mathrm{~g} \mathrm{~mol}^{-1}$. The yield of the pure oligodeoxynucleotide was $513.5 \mu \mathrm{g}(72.1 \mathrm{nmol})$.

The unmodified (hTeloC) and modified (hTeloX) oligonucleotides (ODNs) were dissolved in Milli-Q water to give final concentrations of $100 \mu \mathrm{M}$ and confirmed using a Nanodrop Spectrophotometer (LabTech, Heathfield, UK). The extinction coefficient for the unmodified control sequence (hTeloC) was used to calculate the concentration for both sequences. For all biophysical characterization experiments, ODNs were diluted in buffer containing $10 \mathrm{mM}$ sodium cacodylate and $100 \mathrm{mM}$ sodium chloride at the $\mathrm{pH}$ as detailed, thermally annealed by heating in a heat block at $95{ }^{\circ} \mathrm{C}$ for $5 \mathrm{~min}$, and slowly cooled to room temperature overnight. To allow comparison, all experiments were performed in the same buffer type, though some $\mathrm{pHs}$ examined were outside the buffering capacity of sodium cacodylate $(\mathrm{pH} 4.0,4.5$, and 5.0), and control experiments that examined the $\mathrm{pH}$ under the conditions of the experiment showed little difference in the overall change in pH (see Figure S1).

Circular Dichroism (CD). CD spectra were recorded on a Jasco J-810 spectropolarimeter using a $1 \mathrm{~mm}$ path length quartz cuvette as described previously. ${ }^{21}$ ODNs were diluted to $10 \mu \mathrm{M}$ 
(total volume of $200 \mu \mathrm{L}$ ) in buffer at $\mathrm{pH}$ increments of $0.5 \mathrm{pH}$ unit from $\mathrm{pH} 4.0$ to 8.0. Additional details are included in the Supporting Information. The transitional $\mathrm{pH}\left(\mathrm{pH}_{\mathrm{T}}\right)$ for the imotif was calculated from the inflection point of the fitted ellipticity at $288 \mathrm{~nm}$. Final analysis and manipulation of the data were performed using Origin 2015 (OriginLab, Northhampton, MA).

UV Absorption Spectroscopy. UV spectroscopy experiments were performed as described previously. ${ }^{21}$ Briefly, a Cary $60 \mathrm{UV}-$ visible spectrometer (Agilent Technologies) equipped with a TC1 Temperature Controller (Quantum Northwest) was used to measure the absorbance of a $2.5 \mu \mathrm{M}$ ODN sample at a specific $\mathrm{pH}$ in a small-volume masked quartz cuvette $(1 \mathrm{~cm}$ path length). The absorbance of the ODN was measured at 295 $\mathrm{nm}$ as the temperature of the sample was held for $10 \mathrm{~min}$ at 4 ${ }^{\circ} \mathrm{C}$ and then increased to $95{ }^{\circ} \mathrm{C}$ at a rate of $0.5{ }^{\circ} \mathrm{C} / \mathrm{min}$ and held at $95{ }^{\circ} \mathrm{C}$ for $10 \mathrm{~min}$ before the process was reversed; each melting/annealing process was repeated three times. Data were recorded every $1{ }^{\circ} \mathrm{C}$ during both melting and annealing, and each point was the average of three scans. Melting $\left(T_{\mathrm{m}}\right)$ and annealing $\left(T_{\mathrm{a}}\right)$ temperatures were determined using the firstderivative method. Additional details are available in the Supporting Information. Thermal difference spectra were calculated by subtracting the spectrum between 220 and 320 $\mathrm{nm}$ of the folded structure at $4{ }^{\circ} \mathrm{C}$ from that of the unfolded structure at $95{ }^{\circ} \mathrm{C}$. The data were normalized, and the maximum change in absorption was set to +1 as previously described. $^{22}$

\section{RESULTS AND DISCUSSION}

The $\mathrm{pH}$ and thermal stability of two ODN sequences based on the human telomeric i-motif were examined to determine the effect of GuaUre-dR modification on i-motif formation. The control sequence (hTeloC) remained unmodified, while the modified sequence (hTeloX) included GuaUre-dR instead of cytosine at the last position of the first cytosine tract. We used biophysical techniques to characterize the control and modified sequences; $C D$ was used to determine the $\mathrm{pH}_{\mathrm{T}}$ and $\mathrm{UV}$ spectroscopy to identify the $T_{\mathrm{m}}$ and $T_{\mathrm{a}}$ values under different environmental conditions.

The $\mathrm{pH}$ stabilities of hTeloC and hTeloX sequences were assessed using $\mathrm{CD}$ spectroscopy. The spectra were measured at $\mathrm{pH}$ values ranging from 4 to 8 . At acidic $\mathrm{pH}$ ( $\mathrm{pH} 4.0-5.5)$, the modified and unmodified sequences showed spectra with classic i-motif characteristics: a positive peak at $\sim 288 \mathrm{~nm}$ and a negative peak at $\sim 255 \mathrm{~nm}$ (Figure 3 ). ${ }^{23}$ However, the behavior of the sequences differed at $\mathrm{pH} 6.0$; the hTeloX sequence spectra shifted to more closely resemble that of a random coil with a positive peak at $273 \mathrm{~nm}$ and a negative peak at $250 \mathrm{~nm}$, while the hTeloC spectra remained more similar to those of the i-motif (Figure 3). By pH 6.5, both sequences showed spectra characteristic of random coil, ${ }^{23}$ and the calculated $\mathrm{pH}_{\mathrm{T}}$ values for hTeloX and hTeloC were 5.8 and 6.1, respectively (Figure 3). This indicated that the substitution of GuaUre-dR for a cytosine reduced the $\mathrm{pH}$ stability of the human telomeric imotif-forming sequence.

UV spectroscopy was used to further characterize hTeloC and hTeloX. The thermal stability of each ODN was assessed from $\mathrm{pH} 4.0$ to 7.0. Beyond this $\mathrm{pH}, \mathrm{CD}$ had shown that the sequences would be unfolded; indeed, the $\mathrm{pH} 7.0$ melting and annealing curves could not be normalized for either sequence as they showed no differences in absorbance between high and low temperatures (Figures S2B and S3B and Table S1). At pH
A

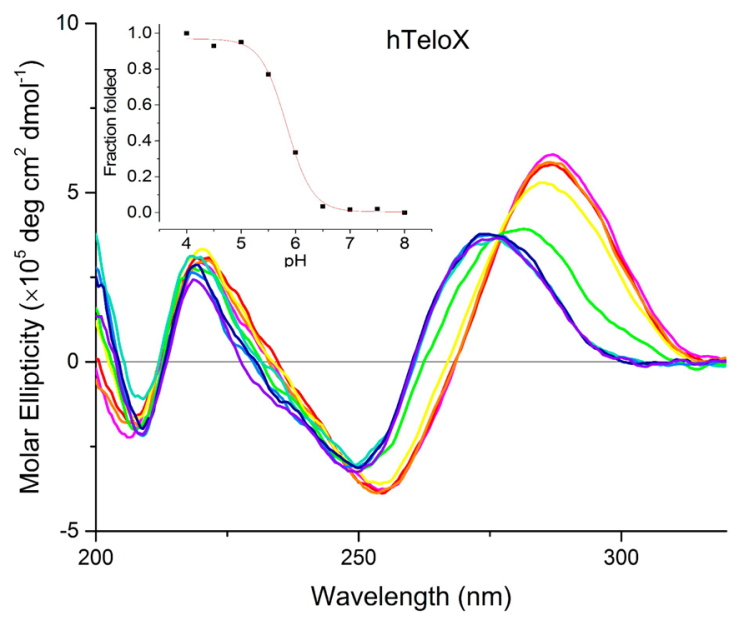

B

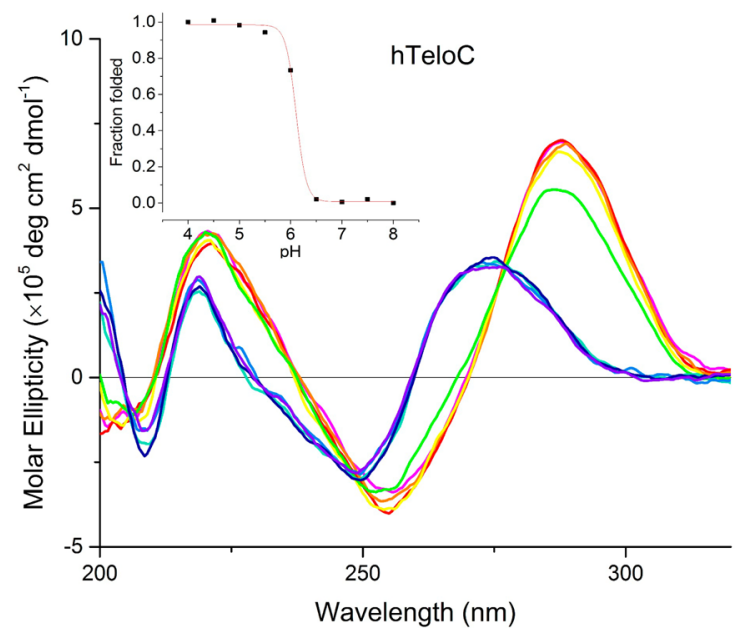

Figure 3. Circular dichroism of (A) the GuaUre-dR-modified hTeloC sequence [the inset shows the fraction folded at $288 \mathrm{~nm}$, used to determine the $\mathrm{pH}_{\mathrm{T}}$ of hTeloX of 5.83 (adjusted $R^{2}=0.997$ )] and (B) the unmodified hTeloC sequence [the inset shows the fraction folded at $288 \mathrm{~nm}$, used to determine the $\mathrm{pH}_{\mathrm{T}}$ of hTeloC of 6.11 (adjusted $R^{2}$

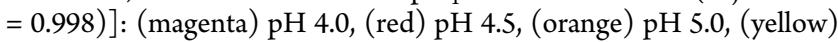
$\mathrm{pH}$ 5.5, (light green) $\mathrm{pH}$ 6.0, (dark green) $\mathrm{pH} 6.5$, (blue) $\mathrm{pH} 7.0$, (black) $\mathrm{pH}$ 7.5, and (purple) $\mathrm{pH}$ 8.0. All oligonucleotide concentrations were $10 \mu \mathrm{M}$ in $10 \mathrm{mM}$ sodium cacodylate buffer with $100 \mathrm{mM}$ sodium chloride buffer at the required $\mathrm{pH}$.

4.0-6.0, both ODNs showed two-state (folded and unfolded) melting and annealing curves (Figures 4, S2, and S3). This was also true of the modified hTeloX at $\mathrm{pH}$ 6.5. However, the unmodified sequence hTeloC showed a three-state UV melting curve at $\mathrm{pH}$ 6.5, resulting in two melting transitions (Figure $4 \mathrm{~A})$. This indicated the possibility of two distinct secondary structures at $\mathrm{pH} 6.5$ and was interesting because the $\mathrm{CD}$ results indicated that at this $\mathrm{pH}$ the secondary structure would be unfolded. A separate study that evaluated the $\mathrm{pH}$ stability of the hTeloC sequence also showed an unfolded $\mathrm{CD}$ spectra at $\mathrm{pH}$ 6.4 and $6.6,{ }^{24}$ which was consistent with the $\mathrm{pH}$ stability reported here. However, only a single value was reported for the $T_{\mathrm{m}}$, suggesting only a single transition in the UV melt at a similar $\mathrm{pH}^{24}$ We have observed double transitions in i-motif sequences previously, ${ }^{21}$ and others have attributed these to an equilibrium mixture composed of the i-motif and hairpin. ${ }^{14,15}$ Here, this isolated phenomenon opens an opportunity for further investigation but does not impact the primary objective 
A

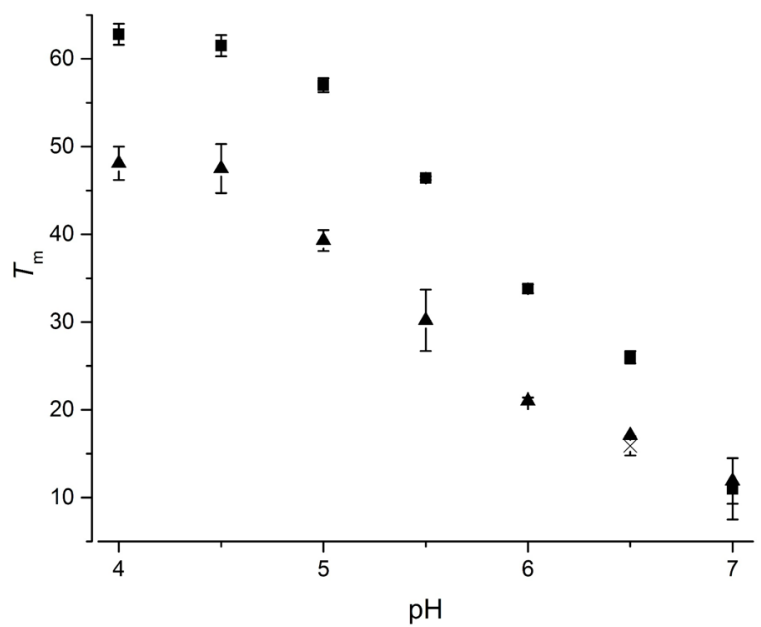

B

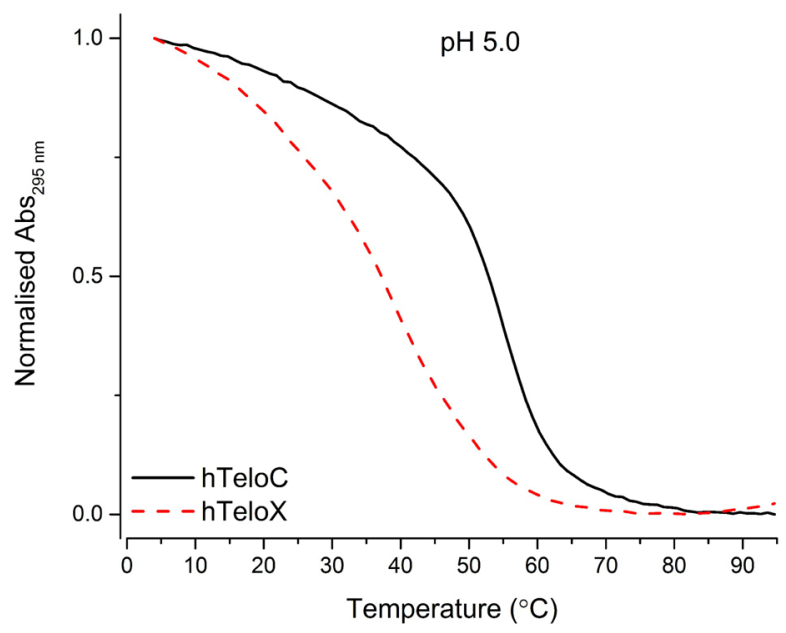

Figure 4. (A) Relationship between the $\mathrm{pH}$ and the $T_{\mathrm{m}}$ of hTeloC and hTeloX. $T_{\mathrm{m}}$ values were determined using the first-derivative method: ( $\left.\boldsymbol{\square}\right)$ hTeloC $T_{\mathrm{m}}$ and $(\boldsymbol{\Delta})$ hTeloX $T_{\mathrm{m}}$. At $\mathrm{pH} 6.5$, the UV melting curve of hTeloC showed two transitions indicating two melting curves. The times sign indicates the secondary melting temperature, $T_{\mathrm{m} 2}$, of hTeloC at this $\mathrm{pH}$. (B) Normalized UV melting curves of GuaUre-dR-modified hTeloX and unmodified hTeloC sequence at $\mathrm{pH}$ 5.0. All oligonucleotide concentrations were $2.5 \mu \mathrm{M}$ in $10 \mathrm{mM}$ sodium cacodylate buffer with $100 \mathrm{mM}$ sodium chloride buffer ( $\mathrm{pH}$ 5.0).

of this work. Increasing the $\mathrm{pH}$ resulted in a decreased thermal stability for both hTeloC and hTeloX (Figure 4A and Table S1). However, hTeloX was less thermally stable than hTeloC; at $\mathrm{pH} 4.0$, the $T_{\mathrm{m}}$ of hTeloC is $15^{\circ} \mathrm{C}$ higher than the $T_{\mathrm{m}}$ for hTeloX (Figure 4A). This difference between the $T_{\mathrm{m}}$ of hTeloC and hTeloX is largest when the absorbance is measured under $\mathrm{pH} 5.0$ conditions $\left[18^{\circ} \mathrm{C}\right.$ (Figure 4B)].

The measurement of thermal difference spectra (TDS) was also used to supplement the characterization of the hTeloC and hTeloX secondary structures. DNA secondary structures absorb UV light differently when they are folded and unfolded, and taking the difference between these spectra gives an indicative spectrum that can be used for characterization. TDS were determined to indicate the dominant folded species of each ODN at each $\mathrm{pH}$. The resultant difference spectra are characteristic of the folded DNA structure present at the lower temperature. ${ }^{22}$ At $\mathrm{pH}$ 4.0-5.0, the TDS of hTeloX showed a positive peak at $240 \mathrm{~nm}$ and a negative peak at 295 $\mathrm{nm}$ (Figure S4). This was consistent with the TDS of the previously characterized folded human telomeric i-motif. ${ }^{22}$ This indicated that the GuaUre-dR-modified sequence (hTeloX) was folded into the i-motif structure between $\mathrm{pH} 4.0$ and 5.5. At higher $\mathrm{pHs}$ (6.0-7.0), the TDS were more indicative of random coil, consistent with the observations using $\mathrm{CD}$ spectroscopy.

Similarly, the TDS of the unmodified hTeloC indicated a folded i-motif. As seen in the UV melting experiment, evidence of a folded i-motif structure (the characteristic positive $240 \mathrm{~nm}$ peak and negative $295 \mathrm{~nm}$ peak) was indicated at all the measured $\mathrm{pH}$ values between 4.0 and 6.5. Only at $\mathrm{pH} 7.0$ does the TDS for hTeloC indicate a random coil structure (Figure S4).

The biophysical evidence presented here indicates that the dominant secondary structure assumed by both the unmodified (hTeloC) and the GuaUre-dR-modified (hTeloX) sequence is an i-motif. The addition of GuaUre-dR instead of a cytosine in the first tract decreases the thermal and $\mathrm{pH}$ stability of the structure, despite its suggested ability to base pair with cytosine. ${ }^{16}$ This degree $\left(10{ }^{\circ} \mathrm{C}\right)$ of thermal destabilization (change in $T_{\mathrm{m}}$ ) is consistent with what others have observed upon mutation of a cytosine, which is known to form C-C base pairs, to a thymine. ${ }^{25}$ This suggests that GuaUre may be interacting with cytosine in a way that does not contribute to imotif stability. It may well be that GuaUre can base pair with a cytosine, just not in the context of an i-motif, but further work would be required to determine whether this is the case.

While GuaUre-dR has been implicated in the overall activity of 5azaC-dR through multiple possible mechanisms, an interaction based on formation of the i-motif is likely more complicated than first thought. DNA secondary structures have been associated with the regulation of genes, and the i-motif in particular has been described as a regulatory switch. ${ }^{14,15}$ Moreover, the cytosine-rich sequences in i-motif-forming regions have been shown to be preferred sites for R-loop formation. ${ }^{26}$ These structures arise when the RNA transcript base pairs with the template strand during transcription, dislodging its DNA complement to form a RNA-DNA hybrid; ${ }^{27}$ the affected regions on the template have no complement on the RNA strand and so form single-stranded bulges. R-Loop formation has been associated with DNA damage and may contribute to genomic instability. ${ }^{28}$ Although the presence of GuaUre-dR in DNA does not inhibit DNA synthesis in vitro, ${ }^{3}$ its effects on RNA synthesis and RNA:DNA hybrid stability are unknown. Even so, it is clear that R-loop formation is confined to regions of GC skew where 5azaC-dR and GuaUre-dR are most likely to be found during drug treatment. Our findings clearly show that GuaUre-dR destabilizes i-motif structures; however, biological consequences of altering the stability of the many structures that can form in cytosine-rich sequences may have additional, and perhaps unforeseen, ramifications in a system as complex as the interaction between $5 \mathrm{azaC}-\mathrm{dR}$ and biological molecules.

\section{ASSOCIATED CONTENT}

Supporting Information

The Supporting Information is available free of charge on the ACS Publications website at DOI: 10.1021/acs.biochem.7b00628. 
Full methodological details, data, and control experiments (PDF)

\section{AUTHOR INFORMATION}

\section{Corresponding Author}

*School of Pharmacy, University of East Anglia, Norwich Research Park, Norwich NR4 7TJ, U.K. E-mail: z.waller@uea. ac.uk. Telephone: +44 (0) 160359 1972. Fax: +44 (0) 160359 2003.

\section{ORCID}

Zoë A. E. Waller: 0000-0001-8538-0484

\section{Funding}

E.P.W. is supported by the Biotechnology and Biological Sciences Research Council (BB/L02229X/1).

\section{Notes}

The authors declare no competing financial interest.

\section{ACKNOWLEDGMENTS}

We thank Dr. Myles Cheesman of the Henry Wellcome Laboratories for Biological Chemistry, University of East Anglia, for the use of the CD spectrometer.

\section{ABBREVIATIONS}

5azaC-dR, 5-aza-2'-deoxycytidine; GuaUre-dR, 2'-deoxyriboguanylurea; hTeloC, human telomeric sequence (5'-TAACCC-TAA-CCC-TAA-CCC-TAA-CCC-3'); hTeloX, GuaUre$\mathrm{dR}(\mathrm{X})$-modified human telomeric sequence (5'-TAA-CCXTAA-CCC-TAA-CCC-TAA-CCC-3'); ODNs, oligonucleotides; $\mathrm{pH}_{\mathrm{T}}$, transitional $\mathrm{pH} ; \mathrm{T}_{\mathrm{a}}$ annealing temperature; TDS, thermal difference spectra; $T_{\mathrm{m}}$, melting temperature.

\section{REFERENCES}

(1) Christman, J. K. (2002) 5-Azacytidine and 5-aza-2'-deoxycytidine as inhibitors of DNA methylation: mechanistic studies and their implications for cancer therapy. Oncogene 21, 5483-5495.

(2) Beisler, J. A., Abbasi, M. M., Kelley, J. A., and Driscoll, J. S. (1977) Synthesis and antitumor activity of dihydro-5-azacytidine, a hydrolytically stable analogue of 5-azacytidine. J. Med. Chem. 20, 806812.

(3) Lamparska, K., Clark, J., Babilonia, G., Bedell, V., Yip, W., and Smith, S. S. (2012) 2'-Deoxyriboguanylurea, the primary breakdown product of 5-aza-2'-deoxyribocytidine, is a mutagen, an epimutagen, an inhibitor of DNA methyltransferases and an inducer of 5-azacytidinetype fragile sites. Nucleic Acids Res. 40, 9788-9801.

(4) Rogstad, D. K., Herring, J. L., Theruvathu, J. A., Burdzy, A., Perry, C. C., Neidigh, J. W., and Sowers, L. C. (2009) Chemical decomposition of 5-aza-2'-deoxycytidine (Decitabine): kinetic analyses and identification of products by NMR, HPLC, and mass spectrometry. Chem. Res. Toxicol. 22, 1194-1204.

(5) Lamparska, K., and Smith, S. S. (2015) The genetic and epigenetic effects of 5-azacytidine and its major breakdown product guanylurea. Epigenetic Diagnosis \& Therapy 1, 28-36.

(6) Ghoshal, K., Datta, J., Majumder, S., Bai, S., Kutay, H., Motiwala, T., and Jacob, S. T. (2005) 5-Aza-deoxycytidine induces selective degradation of DNA methyltransferase 1 by a proteasomal pathway that requires the KEN box, bromo-adjacent homology domain, and nuclear localization signal. Mol. Cell. Biol. 25, 4727-4741.

(7) Smith, S. S. (2013) Maintaining the unmethylated state. Clin. Epigenet. 5, 17-17.

(8) Smith, S. S., Kan, J. L., Baker, D. J., Kaplan, B. E., and Dembek, P. (1991) Recognition of unusual DNA structures by human DNA (cytosine-5)methyltransferase. J. Mol. Biol. 217, 39-51.
(9) Kho, M. R., Baker, D. J., Laayoun, A., and Smith, S. S. (1998) Stalling of human DNA (cytosine-5) methyltransferase at single-strand conformers from a site of dynamic mutation. J. Mol. Biol. 275, 67-79.

(10) Laayoun, A., and Smith, S. S. (1995) Methylation of slipped duplexes, snapbacks and cruciforms by human DNA(cytosine5)methyltransferase. Nucleic Acids Res. 23, 1584-1589.

(11) Chen, X., Mariappan, S. V., Catasti, P., Ratliff, R., Moyzis, R. K., Laayoun, A., Smith, S. S., Bradbury, E. M., and Gupta, G. (1995) Hairpins are formed by the single DNA strands of the fragile X triplet repeats: structure and biological implications. Proc. Natl. Acad. Sci. U. S. A. 92, 5199-5203.

(12) Gehring, K., Leroy, J.-L., and Gueron, M. (1993) A tetrameric DNA structure with protonated cytosine-cytosine base pairs. Nature $363,561-565$.

(13) Day, H. A., Wright, E. P., MacDonald, C. J., Gates, A. J., and Waller, Z. A. E. (2015) Reversible DNA i-motif to hairpin switching induced by copper(II) cations. Chem. Commun. 51, 14099-14102.

(14) Kendrick, S., Kang, H. J., Alam, M. P., Madathil, M. M., Agrawal, P., Gokhale, V., Yang, D., Hecht, S. M., and Hurley, L. H. (2014) The dynamic character of the BCL2 promoter i-motif provides a mechanism for modulation of gene expression by compounds that bind selectively to the alternative DNA hairpin structure. J. Am. Chem. Soc. 136, 4161-4171.

(15) Kang, H. J., Kendrick, S., Hecht, S. M., and Hurley, L. H. (2014) The transcriptional complex between the BCL2 i-motif and hnRNP LL is a molecular switch for control of gene expression that can be modulated by small molecules. J. Am. Chem. Soc. 136, 4172-4185.

(16) Jackson-Grusby, L., Laird, P. W., Magge, S. N., Moeller, B. J., and Jaenisch, R. (1997) Mutagenicity of 5-aza-2'-deoxycytidine is mediated by the mammalian DNA methyltransferase. Proc. Natl. Acad. Sci. U. S. A. 94, 4681-4685.

(17) Phan, A. T., and Mergny, J.-L. (2002) Human telomeric DNA: G-quadruplex, i-motif and Watson-Crick double helix. Nucleic Acids Res. 30, 4618-4625.

(18) Pfleiderer, W. (2007) Universal 2-(4-nitrophenyl)ethyl and 2(4-nitrophenyl)ethoxycarbonyl protecting groups for nucleosides and nucleotides. Current Protocols in Nucleic Acid Chemistry, Chapter 2, Unit 2, 13, Wiley, New York.

(19) Xu, B., Devi, G., and Shao, F. (2015) Regulation of telomeric imotif stability by 5-methylcytosine and 5-hydroxymethylcytosine modification. Org. Biomol. Chem. 13, 5646-5651.

(20) Guimil Garcia, R., Brank, A. S., Christman, J. K., Marquez, V. E., and Eritja, R. (2001) Synthesis of oligonucleotide inhibitors of DNA (cytosine-C5) methyltransferase containing 5-azacytosine residues at specific sites. Antisense Nucleic Acid Drug Dev. 11, 369-378.

(21) Wright, E. P., Huppert, J. L., and Waller, Z. A. E. (2017) Identification of multiple genomic DNA sequences which form i-motif structures at neutral pH. Nucleic Acids Res. 45, 2951-2959.

(22) Mergny, J. L., Li, J., Lacroix, L., Amrane, S., and Chaires, J. B. (2005) Thermal difference spectra: a specific signature for nucleic acid structures. Nucleic Acids Res. 33, e138.

(23) Kypr, J., Kejnovská, I., Renčiuk, D., and Vorlíčková, M. (2009) Circular dichroism and conformational polymorphism of DNA. Nucleic Acids Res. 37, 1713-1725.

(24) Lannes, L., Halder, S., Krishnan, Y., and Schwalbe, H. (2015) Tuning the $\mathrm{pH}$ response of i-motif DNA oligonucleotides. ChemBioChem 16, 1647-1656.

(25) Kendrick, S., Akiyama, Y., Hecht, S. M., and Hurley, L. H. (2009) The i-Motif in the bcl-2 P1 Promoter Forms an Unexpectedly Stable Structure with a Unique 8:5:7 Loop Folding Pattern. J. Am. Chem. Soc. 131, 17667-17676.

(26) Ginno, P. A., Lott, P. L., Christensen, H. C., Korf, I., and Chédin, F. (2012) R-Loop formation is a distinctive characteristic of unmethylated human CpG island promoters. Mol. Cell 45, 814-825.

(27) Groh, M., and Gromak, N. (2014) Out of balance: R-loops in human disease. PLoS Genet. 10, e1004630.

(28) Zeman, M. K., and Cimprich, K. A. (2014) Causes and consequences of replication stress. Nat. Cell Biol. 16, 2-9. 